\title{
Some aspects of nuclear vector groups
}

\author{
by \\ Lydia Aussenhofer (Tübingen)
}

\begin{abstract}
In [2] W. Banaszczyk introduced nuclear groups, a Hausdorff variety of abelian topological groups which is generated by all nuclear vector groups (cf. 2.3) and which contains all nuclear vector spaces and all locally compact abelian groups.

We prove in 5.6 that the Hausdorff variety generated by all nuclear vector spaces and all locally compact abelian groups (denoted by $\mathcal{V}_{1}$ ) is strictly smaller than the Hausdorff variety of all nuclear groups (denoted by $\mathcal{V}_{2}$ ). More precisely, we characterize those nuclear vector groups belonging to $\mathcal{V}_{1}(5.5)$. (These are called special nuclear vector groups.) It is proved that special nuclear vector groups can be embedded into a product of nuclear and of discrete vector spaces (2.5).

The sequence space $\Sigma_{0}$ is introduced (2.6) and it is proved that it is a nuclear but not a special nuclear vector group (2.12). Moreover, together with all discrete vector spaces it generates the Hausdorff variety of all nuclear groups (3.3).

We show that the Hausdorff variety $\mathcal{V}_{0}$ generated by all nuclear vector spaces is strictly contained in $\mathcal{V}_{1}(4.5)$.
\end{abstract}

1. Notation. Let us first fix some notation.

We consider real vector spaces only. For a (real) vector space $E$ and a subset $X \subseteq E$ we denote by $\langle X\rangle_{\mathbb{R}}$ the linear span of $X$ and by $\langle X\rangle_{\mathbb{Z}}$ the subgroup generated by $X$. If $L \subseteq E$ is a linear subspace, we write $L \leq E$. For a normed space $E$, the closed unit ball is denoted by $B_{E}$. Accordingly, if $p$ is a (semi)norm on $E$ then $B_{p}:=\{x \in E: p(x) \leq 1\}$.

$E^{\prime}$ stands for the topological dual of the topological vector space $E$. The polar $X^{0}$ of $X \subseteq E$ is defined by $\left\{\varphi \in E^{\prime}: \varphi(X) \subseteq[-1,1]\right\}$.

For an abelian topological group $G$, the set of neighborhoods of the zero element 0 is denoted by $\mathcal{U}_{G}(0)$.

For a symmetric convex subset $U$ of a vector space $E$, we denote by $p_{U}$ the Minkowski functional of $U$.

$\operatorname{dim} E$ is the cardinality of a basis of $E$ and $|X|$ stands for the cardinality of the set $X . \mathbb{R}^{(X)}$ denotes the subspace of $\mathbb{R}^{X}$ which consists of those families where at most finitely many entries are $\neq 0$.

Finally, $\mathbb{N}=\{1,2,3, \ldots\}$ is the set of natural numbers and $\mathbb{N}_{0}:=\mathbb{N} \cup\{0\}$.

2000 Mathematics Subject Classification: 22A05, 46A11. 
2. (Special) nuclear vector groups. In this section we introduce nuclear vector groups and the subclass of special nuclear vector groups. We will see that the sequence space $\Sigma_{0}$ (defined in 2.6) is a nuclear vector group but not a special nuclear vector group (2.12).

Definition 2.1. For symmetric convex subsets $X, Y$ of a vector space $E$ and $k \in \mathbb{N}$, we put $\mathrm{d}_{k}(X, Y):=\inf \left\{c>0\right.$ : there exists a subspace $L_{c} \leq E$ such that $\operatorname{dim} L_{c}<k$ and $\left.X \subseteq c Y+L_{c}\right\} \in[0, \infty]$.

$\mathrm{d}_{k}(X, Y)$ is called the $k$-th Kolmogorov diameter of $X$ with respect to $Y$ in $E$.

If $T: E_{1} \rightarrow E_{2}$ is a linear operator between the normed spaces $E_{1}, E_{2}$, we set $\mathrm{d}_{k}(T):=\mathrm{d}_{k}\left(T\left(B_{E_{1}}\right), B_{E_{2}}\right)$.

Let us list some properties of the Kolmogorov diameter. One easily verifies that $\mathrm{d}_{k}(T(X), T(Y)) \leq \mathrm{d}_{k}(X, Y)$ for every linear operator $T$. Hence, if $T$ is an isomorphism, then $\mathrm{d}_{k}(T(X), T(Y))=\mathrm{d}_{k}(X, Y)$. For every $c>0$ and all symmetric convex subsets $X, Y$ we have

$$
\mathrm{d}_{k}(c X, Y)=c \mathrm{~d}_{k}(X, Y) \quad \text { and } \quad \mathrm{d}_{k}(X, c Y)=\frac{1}{c} \mathrm{~d}_{k}(X, Y) .
$$

For symmetric convex subsets $X, Y, Z, W$ such that $Z \subseteq X$ and $Y \subseteq W$ we have $\mathrm{d}_{k}(Z, Y) \leq \mathrm{d}_{k}(X, Y)$ and $\mathrm{d}_{k}(X, W) \leq \mathrm{d}_{k}(X, Y)$ for all $k \in \mathbb{N}$.

Recall that a (semi)norm $p$ on a vector space $V$ is named a pre-Hilbert (semi)norm if it satisfies the parallelogram law:

$$
p(x+y)^{2}+p(x-y)^{2}=2 p(x)^{2}+2 p(y)^{2} .
$$

Lemma 2.2. (i) Let $\left(U_{n}\right)_{n \in \mathbb{N}}$ be a sequence of symmetric convex sets in a vector space $E$ satisfying $\mathrm{d}_{k}\left(U_{n+1}, U_{n}\right) \leq 1 / k$ for all $k, n \in \mathbb{N}$. For every $m \in \mathbb{N}$ there exists a subsequence $\left(U_{n_{j}}\right)_{j \in \mathbb{N}}$ such that $n_{1}=1$ and $\mathrm{d}_{k}\left(U_{n_{j+1}}, U_{n_{j}}\right) \leq k^{-m}$ for all $j, k \in \mathbb{N}$.

(ii) For every natural number $m \geq 3$, there exists a constant $\gamma_{m}>0$ such that for all symmetric convex subsets $X, Y$ of a vector space which satisfy $\mathrm{d}_{k}(X, Y) \leq c k^{-m}$ for all $k \in \mathbb{N}$ and a positive constant $c>0$, there are pre-Hilbert seminorms $p$ and $q$ defined on $\langle X\rangle_{\mathbb{R}}$ such that $X \subseteq B_{p}, B_{q} \subseteq Y$, and $\mathrm{d}_{k}\left(B_{p}, B_{q}\right) \leq c \gamma_{m} k^{-m+3}$ for all $k \in \mathbb{N}$.

(iii) Let $p$ be an arbitrary seminorm and let $q$ be a pre-Hilbert seminorm on a vector space $E$ such that $\mathrm{d}_{1}\left(B_{p}, B_{q}\right)<\infty$. For every subspace $E_{0} \leq E$, we have $\mathrm{d}_{k}\left(B_{p} \cap E_{0}, B_{q} \cap E_{0}\right) \leq \mathrm{d}_{k}\left(B_{p}, B_{q}\right)$.

Proof. (i) Cf. (7.1.1) in [9] or 20.2 in [1].

A proof of (ii) can be found in [1] (Lemma 18.32). In [2] (Lemma 2.14), a slightly stronger version is formulated.

(iii) This is Lemma 2.13 of [2] or Lemma 18.18 of [1].

Definitions 2.3. A locally convex Hausdorff vector space $E$ is called a nuclear vector space if for every symmetric convex neighborhood $U \in$ 
$\mathcal{U}_{E}(0)$, there exists a symmetric convex neighborhood $W \in \mathcal{U}_{E}(0)$ such that $\mathrm{d}_{k}(W, U) \leq k^{-1}$ for all $k \in \mathbb{N}$.

A vector space $E$ endowed with a Hausdorff group topology having a 0-neighborhood basis consisting of symmetric convex sets is named a $n u$ clear vector group if it has the following additional property: for every symmetric convex neighborhood $U \in \mathcal{U}_{E}(0)$, there exists a symmetric convex neighborhood $W \in \mathcal{U}_{E}(0)$ such that $\mathrm{d}_{k}(W, U) \leq k^{-1}$ for all $k \in \mathbb{N}$.

We call a nuclear vector group $E$ a special nuclear vector group if there exists a 0 -neighborhood basis $\mathcal{U}_{0}$ consisting of symmetric convex sets such that for every $U_{0} \in \mathcal{U}_{0}$ there exists a sequence $\left(U_{n}\right)_{n \in \mathbb{N}}$ of symmetric convex neighborhoods which satisfy $\left\langle U_{0}\right\rangle_{\mathbb{R}}=\left\langle U_{n}\right\rangle_{\mathbb{R}}$ for all $n \in \mathbb{N}$ and $\mathrm{d}_{k}\left(U_{n}, U_{n-1}\right)$ $\leq 1 / k$ for all $k, n \in \mathbb{N}$.

REMARKS 2.4. (i) The main difference between nuclear vector spaces and nuclear vector groups is that in a nuclear vector group, the linear span $\langle W\rangle_{\mathbb{R}}$ of a smaller neighborhood is in general strictly smaller than $\langle U\rangle_{\mathbb{R}}$.

(ii) In general, a nuclear vector group is not connected. But if it is connected, then every neighborhood of 0 is absorbing. Conversely, if every neighborhood is absorbing, it is easily verified that the scalar multiplication is continuous and hence the nuclear vector group is a topological (and therefore nuclear) vector space.

(iii) For every neighborhood $W$ of 0 in a nuclear vector group and for every $c>0$, the set $c U$ is a neighborhood of 0 .

(iv) It is clear that every nuclear vector space and every discrete vector space (i.e. a vector space endowed with the discrete topology) is a special nuclear vector group.

It follows easily from Lemma 2.7 of [2] that every product of (special) nuclear vector groups is a (special) nuclear vector group. Trivially, every Hausdorff quotient space of a (special) nuclear vector group is a (special) nuclear vector group.

According to 2.2 and (iii), every subspace of a (special) nuclear vector group is a (special) nuclear vector group.

Proposition 2.5. Every special nuclear vector group $E$ can be embedded into a product of nuclear and of discrete vector spaces.

Proof. Fix a neighborhood basis $\mathcal{U}_{0}$ as in the definition. For $U_{0} \in \mathcal{U}_{0}$, we denote by $E_{U_{0}}$ the largest subspace contained in $U_{0}$. As a consequence of $E_{U_{0}} \subseteq\left\{x \in U_{0}: \lambda x \in U_{0} \forall \lambda \in \mathbb{R}\right\} \subseteq p_{U_{0}}^{-1}(0) \subseteq E_{U_{0}}$ and the continuity of $p_{U_{0}}$, we see that $E_{U_{0}}$ is a closed linear subspace. Hence $E / E_{U_{0}}$ is a special nuclear vector group again (2.4(iv)). Let $\psi_{U_{0}}: E \rightarrow E / E_{U_{0}}$ be the canonical projection. 
We fix a sequence $\left(U_{n}\right)_{n \in \mathbb{N}}$ of neighborhoods which satisfies

$$
\mathrm{d}_{k}\left(U_{n}, U_{n-1}\right) \leq 1 / k \quad \forall k, n \in \mathbb{N} \quad \text { and } \quad\left\langle U_{n}\right\rangle_{\mathbb{R}}=\left\langle U_{0}\right\rangle_{\mathbb{R}} \quad \forall n \in \mathbb{N} .
$$

Obviously, the sequence $\left(\psi_{U_{0}}\left(U_{n}\right)\right)_{n \in \mathbb{N}}$ has the corresponding properties in $E / E_{U_{0}}$. So the family $\left(\varepsilon \psi_{U_{0}}\left(U_{n}\right): \varepsilon>0, n \in \mathbb{N}\right)$ forms a 0-neighborhood basis of a nuclear vector space topology on $F_{0}:=\left\langle\psi_{U_{0}}\left(U_{0}\right)\right\rangle_{\mathbb{R}}(2.4(\mathrm{ii})$ ). (Observe further that $\psi_{U_{0}}\left(U_{0}\right)$ contains only the trivial subspace, which implies that this topological space is necessarily Hausdorff.) Let $\widetilde{F}_{U_{0}}$ be an algebraic complement such that $E / E_{U_{0}}=F_{U_{0}} \oplus \widetilde{F}_{U_{0}}$ and let $\pi_{F_{0}}$, respectively $\pi_{\widetilde{F}_{0}}$, denote the corresponding projections onto $F_{U_{0}}$, respectively $\widetilde{F}_{U_{0}}$. Due to the definition, $F_{U_{0}}$ is open in $E / E_{U_{0}}$. We endow $\widetilde{F}_{U_{0}}$ with the discrete topology. Then

$$
\varphi_{U_{0}}: E \rightarrow F_{U_{0}} \times \widetilde{F}_{U_{0}}, \quad x \mapsto\left(\pi_{F_{0}}\left(\psi_{U_{0}}(x)\right), \pi_{\widetilde{F}_{U_{0}}}\left(\psi_{U_{0}}(x)\right)\right),
$$

is a well defined, continuous linear mapping with kernel $E_{U_{0}}$; moreover $\varphi_{U_{0}}\left(U_{0}\right)$ is a neighborhood of 0 . So

$$
\varphi: E \rightarrow \prod_{U_{0} \in \mathcal{U}_{0}} F_{U_{0}} \times \widetilde{F}_{U_{0}}, \quad x \mapsto\left(\varphi_{U_{0}}(x)\right)_{U_{0} \in \mathcal{U}_{0}}
$$

is a continuous monomorphism. Since

$$
\varphi\left(\widetilde{U}_{0}\right)=\varphi(E) \cap\left(\left(\prod_{U_{0} \in \mathcal{U}_{0}, U_{0} \neq \widetilde{U}_{0}} F_{U_{0}} \times \widetilde{F}_{U_{0}}\right) \times \varphi_{\widetilde{U}_{0}}\left(\widetilde{U}_{0}\right)\right)
$$

for every $\widetilde{U}_{0} \in \mathcal{U}_{0}, \varphi$ is an embedding.

Next we present a concrete example of a nuclear vector group.

ExAmple 2.6. For $r \in \mathbb{N}_{0}$, let

$$
p_{r}: \Sigma_{r} \rightarrow \mathbb{R}, \quad\left(x_{n}\right)_{n \in \mathbb{N}} \mapsto \sup \left\{n^{r}\left|x_{n}\right|: n \in \mathbb{N}\right\},
$$

where

$$
\Sigma_{r}:=\left\{\left(x_{n}\right)_{n \in \mathbb{N}} \in \mathbb{R}^{\mathbb{N}}:\left(n^{r} x_{n}\right)_{n \in \mathbb{N}} \text { is bounded }\right\} .
$$

For $\Sigma:=\bigcap_{r \in \mathbb{N}} \Sigma_{r}$ we have

$$
\mathrm{d}_{k}\left(B_{p_{r+1}}, B_{p_{r}}\right) \leq k^{-1} \quad \text { and } \quad \mathrm{d}_{k}\left(B_{p_{r+1}} \cap \Sigma, B_{p_{r}} \cap \Sigma\right) \leq k^{-1} .
$$

[Observe that $B_{p_{r+1}} \subseteq k^{-1} B_{p_{r}}+\left\langle e_{1}, \ldots, e_{k-1}\right\rangle_{\mathbb{R}}$ (where $\left(e_{j}\right)_{j \in \mathbb{N}}$ denotes the sequence of the standard unit vectors); the analogous inclusions hold for the intersection with $\Sigma$.]

We endow the vector space $\Sigma_{0}:=\mathbb{R}^{\mathbb{N}}$ with the group topology induced by the neighborhoods $\left(\varepsilon B_{p_{r}}: r \in \mathbb{N}, \varepsilon>0\right)$ and observe that it is a nuclear vector group. $\Sigma$ endowed with the topology induced by the norms $\left.p_{r}\right|_{\Sigma}$ is a nuclear vector space. Moreover, it is easily verified that $\Sigma$ is the connected component of 0 in $\Sigma_{0}$. 
Furthermore, for $r \in \mathbb{N}_{0}$, we define $V_{r}:=\left\{\left(x_{n}\right)_{n \in \mathbb{N}}: \sum_{n \in \mathbb{N}}\left(n^{r} x_{n}\right)^{2}<\infty\right\}$ and

$$
q_{r}: V_{r} \rightarrow \mathbb{R}, \quad\left(\left(x_{n}\right)_{n \in \mathbb{N}}\right) \mapsto \sqrt{\sum_{n \in \mathbb{N}}\left(n^{r} x_{n}\right)^{2}} .
$$

It follows easily that

$$
V_{r} \subseteq \Sigma_{r} \subseteq V_{r-1}, \quad q_{r} \geq\left. p_{r}\right|_{V_{r}}, \quad p_{r} \geq\left.\frac{\sqrt{6}}{\pi} q_{r-1}\right|_{\Sigma_{r}}
$$

for all $r \in \mathbb{N}$. Hence the family $\left(\varepsilon B_{q_{r}}: \varepsilon>0, r \in \mathbb{N}_{0}\right)$ generates the given topology on $\Sigma_{0}$ and for every $r \in \mathbb{N}_{0},\left(V_{r}, q_{r}\right)$ is a Hilbert space.

Lemma 2.7. Let $T: H_{1} \rightarrow H_{2}$ be a continuous linear operator between inner product spaces. If $T^{\prime}$ denotes the adjoint operator, then $\mathrm{d}_{k}\left(T^{\prime}\right)=$ $\mathrm{d}_{k}(T)$ for all $k \in \mathbb{N}$.

Proof. The proof is straightforward. Alternatively, it is a consequence of (11.7.6) and (11.3.4) in [8] and of (18.16) in [1].

LEMMA 2.8. For every normed space $E$ and every finite-dimensional subspace $L \leq E$, the sum of the closed unit ball $B$ with $L$ is closed.

Proof. This is an easy consequence of the Bolzano-Weierstraß theorem.

Lemma 2.9. Let $T: H_{1} \rightarrow H_{2}, x \mapsto \sum_{j \in \mathbb{N}} \alpha_{j}\left(x, x_{j}\right)_{1} \cdot y_{j}$, be a continuous linear operator between the Hilbert spaces $\left(H_{1},(\cdot, \cdot)_{1}\right)$ and $\left(H_{2},(\cdot, \cdot)_{2}\right)$, where $\left(x_{j}\right)_{j \in \mathbb{N}}$ and $\left(y_{j}\right)_{j \in \mathbb{N}}$ are orthonormal systems in $H_{1}$ and $H_{2}$ respectively and $\left(\alpha_{j}\right)_{j \in \mathbb{N}}$ is a decreasing sequence of non-negative real numbers. Then $\mathrm{d}_{k}(T)=\alpha_{k}$ for all $k \in \mathbb{N}$.

Proof. This is Proposition (11.3.3) in [8].

We intend to apply these results to the sequence space $\Sigma_{0}$; we use the notation introduced in 2.6.

Lemma 2.10. For every $r \in \mathbb{N}_{0}$ and all $k \in \mathbb{N}$ we have $\mathrm{d}_{k}\left(B_{q_{r}}, B_{q_{0}}\right)=k^{-r}$.

Proof. Since $\left(k^{-r} e_{k}: k \in \mathbb{N}\right)$ is an orthonormal basis of $\left(V_{r}, q_{r}\right)$, the inclusion $V_{r} \rightarrow V_{0}$ can be written in the form

$$
\left(x_{n}\right)=\sum_{k \in \mathbb{N}}\left(\left(x_{n}\right), k^{-r} e_{k}\right)_{r} \cdot k^{-r} e_{k}=\sum_{k \in \mathbb{N}} k^{-r}\left(\left(x_{n}\right), k^{-r} e_{k}\right)_{r} \cdot e_{k} .
$$

(The scalar product associated with $q_{r}$ is denoted by $\left.(\cdot, \cdot)_{r}.\right)$ It follows from 2.9 that $\mathrm{d}_{k}\left(B_{q_{r}}, B_{q_{0}}\right)=k^{-r}$ for all $k \in \mathbb{N}$.

Lemma 2.11. For every $r \in \mathbb{N}_{0}$ and every symmetric, convex, absorbing subset $X \subseteq V_{r}$, there exists $\varepsilon>0$ such that $\mathrm{d}_{k}\left(X, B_{q_{0}}\right) \geq \varepsilon k^{-r}$ for all $k \in \mathbb{N}$.

Proof. Fix $r \in \mathbb{N}_{0}$. Since $V_{r}=\bigcup_{n \in \mathbb{N}} n \bar{X}^{q_{r}}$ and $\left(V_{r}, q_{r}\right)$ is a Hilbert space, in particular a Baire space, there exists $n \in \mathbb{N}$ such that the interior of $n \bar{X}^{q_{r}}$ 
is not empty. It follows easily that $\bar{X}^{q_{r}} \supseteq \varepsilon B_{q_{r}}$ for suitable $\varepsilon>0$. (The norm as upper index denotes with respect to which topology the closure is taken.)

Next we prove that $\mathrm{d}_{k}\left(X, B_{q_{0}}\right)=\mathrm{d}_{k}\left(\bar{X}^{q_{r}}, B_{q_{0}}\right)$. The inequality " $\leq$ " is trivial. Conversely, let $c>0$, and $L \leq \Sigma_{0}$ be of finite dimension such that $X \subseteq c B_{q_{0}}+L$. Without loss of generality we may assume that $L \leq V_{0}$. Then $\bar{X}^{q_{r}} \subseteq{\overline{\left(c B_{q_{0}}+L\right) \cap V_{r}}}_{q_{r}} \subseteq{\overline{c B_{q_{0}}+L}}^{q_{0}} \stackrel{2.8}{=} c B_{q_{0}}+L$, which implies " $\geq$ ".

Combining these results yields

$$
\mathrm{d}_{k}\left(X, B_{q_{0}}\right)=\mathrm{d}_{k}\left(\bar{X}^{q_{r}}, B_{q_{0}}\right) \geq \mathrm{d}_{k}\left(\varepsilon B_{q_{r}}, B_{q_{0}}\right)=\varepsilon \mathrm{d}_{k}\left(B_{q_{r}}, B_{q_{0}}\right) \stackrel{2.10}{=} \varepsilon k^{-r}
$$

for all $k \in \mathbb{N}$, which was to be shown.

THEOREM 2.12. The nuclear vector group $\Sigma_{0}$ is not a special nuclear vector group.

Proof. Suppose the converse. This means: there exists a sequence $\left(U_{n}\right)_{n \in \mathbb{N}_{0}}$ of symmetric convex neighborhoods which satisfies:

(i) $U_{0} \subseteq B_{q_{0}}$,

(ii) $\mathrm{d}_{k}\left(U_{n+1}, U_{n}\right) \leq 1 / k$ for all $k \in \mathbb{N}$ and $n \in \mathbb{N}_{0}$, and

(iii) $\left\langle U_{n}\right\rangle_{\mathbb{R}}=\left\langle U_{0}\right\rangle_{\mathbb{R}}$ for all $n \in \mathbb{N}$.

Applying 2.2(i) inductively enables us to find a subsequence of $\left(U_{n}\right)_{n \in \mathbb{N}}$ (again denoted by $\left(U_{n}\right)_{n \in \mathbb{N}}$ ) which satisfies

(ii) ${ }^{\prime} \mathrm{d}_{k}\left(U_{n}, U_{0}\right) \leq k^{-n}$ for all $k, n \in \mathbb{N}$.

There exists $r \in \mathbb{N}$ such that $V_{r} \subseteq\left\langle U_{0}\right\rangle_{\mathbb{R}}$. Hence we get

$$
\mathrm{d}_{k}\left(U_{n} \cap V_{r}, B_{q_{0}}\right) \stackrel{\text { (i) }}{\leq} \mathrm{d}_{k}\left(U_{n}, U_{0}\right) \stackrel{\text { (ii) }^{\prime}}{\leq} k^{-n} \quad \text { for all } k, n \in \mathbb{N} .
$$

Since the $U_{n} \cap V_{r}$ satisfy the assumptions of 2.11 , there is a sequence $\left(\varepsilon_{n}\right)_{n \in \mathbb{N}}$ of positive numbers such that

$$
\varepsilon_{n} k^{-r} \leq \mathrm{d}_{k}\left(U_{n} \cap V_{r}, B_{q_{0}}\right) \leq k^{-n} \quad \forall k, n \in \mathbb{N} .
$$

This contradiction completes the proof.

3. Embedding of nuclear vector groups. We want to show in this section that every nuclear vector group can be embedded into a product of copies of $\Sigma_{0}$ and of discrete vector spaces.

Lemma 3.1. Every nuclear vector group $E$ has a 0 -neighborhood basis $\mathcal{U}_{0}$ consisting of symmetric convex sets such that for every $W \in \mathcal{U}_{0}$ there exists a sequence $\left(\varphi_{n}\right)_{n \in \mathbb{N}}$ of linear forms defined on $\langle W\rangle_{\mathbb{R}}$ which satisfies:

(i) $\left(n^{r} \varphi_{n}\right)_{n \in \mathbb{N}}$ is equicontinuous for every $r \in \mathbb{N}$ and

(ii) $\left\{x \in\langle W\rangle_{\mathbb{R}}:\left|\varphi_{n}(x)\right| \leq(2 n)^{-1} \forall n \in \mathbb{N}\right\} \subseteq W$. 
Proof. Let $U$ be a symmetric convex neighborhood of 0 . We have to show that there exists a symmetric convex neighborhood $W$ of 0 , contained in $U$, which has the properties stated above.

We may assume that $U$ contains only the trivial subspace.

$\left[p_{U}^{-1}(0)=\{x \in E: \lambda x \in U \forall \lambda \in \mathbb{R}\}\right.$ is a closed linear subspace, and hence $E / p_{U}^{-1}(0)$ is a nuclear vector group again. Let $\pi: E \rightarrow E / p_{U}^{-1}(0)$ be the canonical projection. Then $\pi(U)$ contains only the trivial subspace. If we find $\widetilde{W} \subseteq \pi(U)$ and $\left(\widetilde{\varphi}_{n}\right)$ which satisfy the assertions in the quotient space, then $\pi^{-1}(\widetilde{W})$ and $\varphi_{n}:=\widetilde{\varphi}_{n} \circ \pi$ have the desired properties in $E$.]

According to 2.2 , there is a sequence $s_{n}: E_{n} \rightarrow \mathbb{R}\left(n \in \mathbb{N}_{0}\right)$ of continuous pre-Hilbert norms defined on a decreasing sequence $\left(E_{n}\right)_{n \in \mathbb{N}_{0}}$ of subspaces such that

$$
B_{s_{0}} \subseteq U \quad \text { and } \quad \mathrm{d}_{k}\left(B_{s_{n}}, B_{s_{0}}\right) \leq k^{-2 n} \quad \text { for all } k \in \mathbb{N}, n \in \mathbb{N}_{0} .
$$

Moreover, we may assume that $E_{0}=E_{1}$. [Replacing $E_{0}$ by $E_{1}$ and $s_{0}$ by $\left.s_{0}\right|_{E_{1}}$ implies that $B_{\left.s_{0}\right|_{E_{1}}}=B_{s_{0}} \cap E_{1}$ is a neighborhood of 0 and

$$
\left.\mathrm{d}_{k}\left(B_{\left.s_{0}\right|_{E_{1}}}, B_{s_{1}}\right)=\mathrm{d}_{k}\left(B_{s_{0}} \cap E_{1}, B_{s_{1}}\right) \leq \mathrm{d}_{k}\left(B_{s_{0}}, B_{s_{1}}\right) \cdot\right]
$$

For $n \in \mathbb{N}$, let $T_{n}:\left(E_{n}, s_{n}\right) \rightarrow\left(E_{0}, s_{0}\right)$ be the inclusion map. For the adjoint operator $T_{n}^{\prime}:\left(E_{0}, s_{0}\right)^{\prime} \rightarrow\left(E_{n}, s_{n}\right)^{\prime}$ we have

$$
\mathrm{d}_{k}\left(T_{n}^{\prime}\right) \stackrel{2.7}{=} \mathrm{d}_{k}\left(T_{n}\right)=\mathrm{d}_{k}\left(B_{s_{n}}, B_{s_{0}}\right) \stackrel{(1)}{\leq} k^{-2 n} \quad \text { for all } k, n \in \mathbb{N} .
$$

According to (9.1.4) of [7], $T_{n}^{\prime}$ is a compact operator for all $n \in \mathbb{N}$.

If one of the spaces $E_{n}$ has finite dimension, then the assertion is clear, since the $E_{n}$ are open in $E$ and the weak topology on a finite-dimensional vector space coincides with the given one. So let us assume the converse.

Due to the spectral decomposition theorem (cf. (8.3.1) in [7]), for all $n \in \mathbb{N}$ there are orthonormal systems $\left(\varphi_{n, k}\right)_{k \in \mathbb{N}}$ in $\left(E_{0}, s_{0}\right)^{\prime}$ and $\left(\psi_{n, k}\right)_{k \in \mathbb{N}}$ in $\left(E_{n}, s_{n}\right)^{\prime}$ and a decreasing sequence $\left(\alpha_{n, k}\right)_{k \in \mathbb{N}}$ of non-negative numbers such that

$$
T_{n}^{\prime}(\varphi)=\sum_{k \in \mathbb{N}} \alpha_{n, k}\left(\varphi, \varphi_{n, k}\right)_{0} \cdot \psi_{n, k} \quad \forall n \in \mathbb{N}, \forall \varphi \in\left(E_{0}, s_{0}\right)^{\prime}
$$

where $(\cdot, \cdot)_{n}$ denotes the inner product of the Hilbert space $\left(E_{n}, s_{n}\right)^{\prime}$ (for $n \in \mathbb{N}_{0}$ ). Furthermore, according to 2.9 and (2), we have

$$
\alpha_{n, k}=\mathrm{d}_{k}\left(T_{n}^{\prime}\right) \leq k^{-2 n} .
$$

Since $T_{1}$ is surjective, the adjoint operator $T_{1}^{\prime}$ is injective and hence $\left(\varphi_{1, k}\right)_{k \in \mathbb{N}}$ is an orthonormal basis of $\left(E_{0}, s_{0}\right)^{\prime}$. Hence the closed linear span of the set $\left\{\varphi_{n, k}: n, k \in \mathbb{N}\right\}$ equals $\left(E_{0}, s_{0}\right)^{\prime}$. We form the Gram-Schmidt 
"orthonormalization" of the $\varphi_{n, k}$ with respect to $(\cdot, \cdot)_{0}$ in the following order:

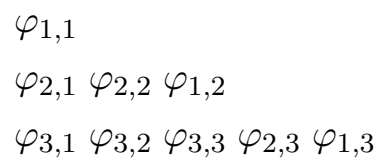

Observe that the family $\left(\varphi_{n, k}: n, k \in \mathbb{N}\right)$ need not be linearly independent. (In this case it happens that some $\varphi_{l}$ are 0 and hence not of norm 1.) Anyhow, the set $\left\{\varphi_{l}: l \in \mathbb{N}\right\} \backslash\{0\}$ forms an orthonormal basis of $\left(E_{0}, s_{0}\right)^{\prime}$.

Since the $k$-th row has $2 k-1$ entries and $\varphi_{n, k}$ appears in the $\max (n, k)$-th row, we get

$$
\left(\varphi_{l}, \varphi_{n, k}\right)_{0}=0 \quad \text { for } l>\sum_{j=1}^{\max (n, k)}(2 j-1)=\max (n, k)^{2} .
$$

Moreover, putting $\varphi_{l}$ in (3) yields

$$
T_{n}^{\prime}\left(\varphi_{l}\right)=\left.\varphi_{l}\right|_{E_{n}}=\sum_{k \geq \sqrt{l}} \alpha_{n, k}\left(\varphi_{l}, \varphi_{n, k}\right)_{0} \cdot \psi_{n, k} \quad \text { for all } l>n^{2}
$$

and hence for all $l>n^{2}$ we have

$$
\begin{aligned}
\left(\varphi_{l}\left|E_{n}, \varphi_{l}\right|_{E_{n}}\right)_{n} & \stackrel{(5)}{=} \sum_{k \geq \sqrt{l}}\left(\alpha_{n, k}\left(\varphi_{l}, \varphi_{n, k}\right)_{0}\right)^{2} \stackrel{(4)}{\leq} \sum_{k \geq \sqrt{l}} k^{-4 n}\left(\left(\varphi_{l}, \varphi_{n, k}\right)_{0}\right)^{2} \\
& \leq l^{-2 n} \sum_{k \geq \sqrt{l}}\left(\left(\varphi_{l}, \varphi_{n, k}\right)_{0}\right)^{2} \leq l^{-2 n}
\end{aligned}
$$

by the Bessel inequality. This implies $l^{n} \varphi_{l} \in B_{s_{n}}^{0}$ for all $l>n^{2}$ and hence the set $\left\{l^{r} \varphi_{l}: l \in \mathbb{N}\right\}$ is equicontinuous for all $r \in \mathbb{N}$.

Now, let $x \in E_{0}$ satisfy $\left|\varphi_{n}(x)\right| \leq 1 /(2 n)$ for all $n \in \mathbb{N}$ and let $\varphi \in B_{s_{0}}^{0}$. Since $\varphi$ can be represented in the form $\varphi=\sum_{l \in \mathbb{N}}\left(\varphi, \varphi_{l}\right)_{0} \cdot \varphi_{l}$, we get

$$
|\varphi(x)| \leq \sqrt{\sum_{l \in \mathbb{N}}\left(\left(\varphi, \varphi_{l}\right)_{0}\right)^{2}} \sqrt{\sum_{l \in \mathbb{N}} \varphi_{l}(x)^{2}} \leq(\varphi, \varphi)_{0} \sqrt{\frac{\pi^{2}}{4 \cdot 6}}<1 .
$$

By the Hahn-Banach theorem, $x$ is an element of $B_{s_{0}}$ and hence $W:=B_{s_{0}}$ and $\left(\varphi_{l}\right)_{l \in \mathbb{N}}$ have the desired properties.

COROLlary 3.2. With the notation introduced above, for every $W \in \mathcal{U}_{0}$ there exists a continuous linear mapping $\Phi_{W}:\langle W\rangle_{\mathbb{R}} \rightarrow \Sigma_{0}$ which satisfies $W \supseteq \Phi_{W}^{-1}\left(\frac{1}{2} B_{p_{1}}\right)\left(B_{p_{1}}\right.$ as in 2.6); in particular, $\Phi_{W}(x) \neq 0$ for all $x \in$ $\langle W\rangle_{\mathbb{R}} \backslash W$. 
Proof. Let $\left(\varphi_{n}\right)_{n \in \mathbb{N}}$ have the properties stated in 3.1 and put

$$
\Phi_{W}:\langle W\rangle_{\mathbb{R}} \rightarrow \Sigma_{0}, \quad x \mapsto\left(\varphi_{n}(x)\right)_{n \in \mathbb{N}} .
$$

It is clear that $\Phi_{W}$ is a linear operator.

Fix $r \in \mathbb{N}$. By the equicontinuity of the sequence $\left(n^{r} \varphi_{n}\right)_{n \in \mathbb{N}}$, there exists a neighborhood $U_{r}$ of 0 in $\langle W\rangle_{\mathbb{R}}$ such that $\varphi_{n}(x) \leq n^{-r}$ for all $x \in U_{r}$. Hence $\Phi_{W}\left(U_{r}\right) \subseteq B_{p_{r}}$ and the continuity of $\Phi_{W}$ follows.

According to 3.1(ii), $W \supseteq \Phi_{W}^{-1}\left(\frac{1}{2} B_{p_{1}}\right)$, which implies $\Phi_{W}(x) \neq 0$ for all $x \in\langle W\rangle_{\mathbb{R}} \backslash W$.

TheOREM 3.3. Every nuclear vector group $E$ can be embedded into a product of copies of $\Sigma_{0}$ and of discrete vector spaces.

Proof. Let $\mathcal{U}_{0}$ be a neighborhood basis having the properties stated in 3.1. For $W \in \mathcal{U}_{0}$, we fix a complementary subspace $E_{W}$ such that $E=$ $\langle W\rangle_{\mathbb{R}} \oplus E_{W}$ (algebraically). Endowing $E_{W}$ with the discrete topology makes the above decomposition topological. Let $\pi_{W}: E \rightarrow\langle W\rangle_{\mathbb{R}}$ denote the projection with kernel $E_{W}$ and for $\Phi_{W}$ as in 3.2, put

$$
\Phi: E \rightarrow \prod_{W \in \mathcal{U}_{0}}\left(\Sigma_{0} \times E_{W}\right), \quad x \mapsto\left(\Phi_{W}\left(\pi_{W}(x)\right), x-\pi_{W}(x)\right)_{W \in \mathcal{U}_{0}} .
$$

According to $3.2, \Phi$ is a continuous monomorphism and

$$
\Phi(W) \supseteq \operatorname{im} \Phi \cap\left(\left(\frac{1}{2} B_{p_{1}} \times\{0\}\right) \times \prod_{W \neq W^{\prime} \in \mathcal{U}_{0}}\left(\Sigma_{0} \times E_{W^{\prime}}\right)\right)
$$

for all $W \in \mathcal{U}_{0}$. [Observe that an element of the right hand side is of the form $\Phi(x)$ where $x \in\langle W\rangle_{\mathbb{R}} ;$ moreover, $\Phi_{W}(x) \in \frac{1}{2} B_{p_{1}}$ implies $x \in W$.] Hence $\Phi$ is an embedding.

Corollary 3.4 (T. and Y. Kōmura). Every nuclear vector space E can be embedded into a product of copies of $\Sigma$.

Proof. We use the notation introduced in the proof of 3.3. One has to observe that every neighborhood of 0 in $E$ is absorbing, which implies that $E_{W}=\{0\}$ for every $W \in \mathcal{U}_{0}$. Moreover, $\Phi_{W}(E)$ is a topological vector space and a subspace of $\Sigma_{0}$, hence contained in the component $\Sigma$ (see 2.4).

This corollary was first proved by T. and Y. Kōmura in [5].

\section{On the variety generated by all nuclear vector spaces}

Notation 4.1. Let $\mathcal{C}$ be a class of topological groups. By $\mathcal{V}(\mathcal{C})$ we denote the smallest class of topological groups which contains $\mathcal{C}$ and which is closed under forming arbitrary products, Hausdorff quotient groups, arbitrary subgroups, and which is closed under topological isomorphisms. Then $\mathcal{V}(\mathcal{C})$ is called the Hausdorff (group-) variety generated by $\mathcal{C}$. 
It is well known that every member of $\mathcal{V}(\mathcal{C})$ is topologically isomorphic to a Hausdorff quotient group of a subgroup of a product of elements of $\mathcal{C}$ (cf. [4], p. 65). In particular, $\mathcal{V}(\mathcal{C})$ exists. If $\mathcal{C}$ consists of abelian Hausdorff groups, so does $\mathcal{V}(\mathcal{C})$.

In what follows we use the following abbreviations:

- $\mathcal{V}_{0}$ for the Hausdorff variety generated by all nuclear vector spaces,

- $\mathcal{V}_{1}$ for the Hausdorff variety generated by all nuclear vector spaces and all discrete abelian groups, and

- $\mathcal{V}_{2}$ for the Hausdorff variety generated by all nuclear vector groups.

In this section we are going to show that $\mathcal{V}_{0}$ is a proper subclass of $\mathcal{V}_{1}$.

LEMMA 4.2. Let $E$ be a nuclear vector space which has a neighborhood $U \in \mathcal{U}_{E}(0)$ containing only the trivial subspace. Then $\operatorname{dim} E \leq|\mathbb{R}|$.

Proof. Choose linear functionals $\varphi_{n}: E \rightarrow \mathbb{R}$ as in 3.1 associated with a neighborhood $W$ contained in $U$. Since $W$ contains only the trivial subspace, we conclude from 3.1(ii) that $\Phi:=\left(\varphi_{n}\right): E \rightarrow \mathbb{R}^{\mathbb{N}}$ is injective. Hence $\operatorname{dim} E \leq \operatorname{dim} \mathbb{R}^{\mathbb{N}} \leq\left|\mathbb{R}^{\mathbb{N}}\right|=|\mathbb{R}|$

Lemma 4.3. For a symmetric convex subset $C$ of a vector space $E$ and a subgroup $H$ of $E$, put $U:=H \cap C, H_{U}:=\left\{x \in H:\langle x\rangle_{\mathbb{Z}} \subseteq U\right\}$, and $E_{C}:=\left\{x \in E:\langle x\rangle_{\mathbb{R}} \subseteq C\right\}$. Then $H_{U}$ is the largest subgroup contained in $U, E_{C}$ is the largest subgroup (subspace) contained in $C$, and $E_{C} \cap H=H_{U}$. in $C$.

Proof. Since $E_{C}=p_{C}^{-1}(0), E_{C}$ is the largest linear subspace contained

Obviously, $E_{C} \cap H \subseteq H_{U}$. Conversely, for $x \in H_{U}$, we have $\langle x\rangle_{\mathbb{Z}} \subseteq U \subseteq C$ and hence $p_{C}(k x) \leq 1$ for all $k \in \mathbb{Z}$. This implies $p_{C}(x)=0$ and hence $x \in E_{C} \cap H$. Combining these inclusions yields $E_{C} \cap H=H_{U}$.

This shows that $H_{U}$ is a subgroup of $H$ contained in $U$; by the definition of $H_{U}$, it is the largest one.

Using the above notation, we get the following commutative square where all mappings are the canonical ones and where $\tau$ is injective.

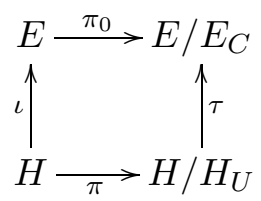

TheOREM 4.4. A discrete abelian group $D$ does not belong to $\mathcal{V}_{0}$ if $|D|>$ $|\mathbb{R}|$.

Proof. For $D \in \mathcal{V}_{0}$, there exists a nuclear vector space $E$, a subgroup $H \leq E$ and a projection $\varphi: H \rightarrow D$. Since $D$ is discrete, there is a continuous seminorm $p$ on $E$ such that $\varphi\left(H \cap B_{p}\right)=\{0\}$. Put $U:=H \cap B_{p}$ and $C:=B_{p}$. 
Since $\varphi$ induces an epimorphism $\widetilde{\varphi}: H / H_{U} \rightarrow D$, we get $|D| \leq\left|H / H_{U}\right| \leq$ $\left|E / E_{C}\right|$ (by the injectivity of $\tau$ ). According to 4.2 , the nuclear vector space $E / E_{C}$ is of dimension $\leq|\mathbb{R}|$ and hence $\left|E / E_{C}\right| \leq\left|\mathbb{R}^{(\mathbb{R})}\right|=|\mathbb{R}|$. This implies the assertion.

COROllary 4.5. The variety $\mathcal{V}_{0}$ is properly contained in $\mathcal{V}_{1}$

\section{On the variety generated by all nuclear groups}

REMARK 5.1. In [2], nuclear groups have been introduced and it is shown there that the class of nuclear groups forms a Hausdorff variety (Chapter 7) which coincides with $\mathcal{V}_{2}$ (Theorem 9.6 of [2]).

As a consequence of the structure theorem for locally compact abelian groups, these groups are nuclear (7.10 in [2]); even more, the same argument shows that they belong to the (possibly) smaller variety $\mathcal{V}_{1}$. Of course, nuclear vector spaces are also nuclear groups. Since these examples all belong to $\mathcal{V}_{1}$, the question arose whether every nuclear group belonged to $\mathcal{V}_{1}$.

In this section we show that $\mathcal{V}_{1}$ is a proper subclass of $\mathcal{V}_{2}$.

Lemma 5.2. For every subgroup $H$ of a nuclear vector group $(E, \mathcal{O})$, the sets $(\operatorname{conv} U)_{U \in \mathcal{U}_{H}(0)}$ form a 0-neighborhood basis of a nuclear vector group topology $\mathcal{O}_{\text {conv }}$; the inclusion $H \rightarrow\left(E, \mathcal{O}_{\text {conv }}\right)$ is an embedding and the identity mapping $\left(E, \mathcal{O}_{\text {conv }}\right) \rightarrow(E, \mathcal{O})$ is continuous.

Proof. For $U^{\prime}, U \in \mathcal{U}_{H}(0)$ such that $U^{\prime}+U^{\prime} \subseteq U$ we get $U^{\prime} \subseteq \frac{1}{2} \operatorname{conv} U$ and hence conv $U^{\prime}+\operatorname{conv} U^{\prime} \subseteq \operatorname{conv} U$. Now it is easy to verify that the sets (conv $U: U \in \mathcal{U}_{H}(0)$ ) form a neighborhood basis of a group topology $\mathcal{O}_{\text {conv }}$ on $E$. Since for every symmetric convex neighborhood $U_{E} \in \mathcal{U}_{E}(0)$, we have $\operatorname{conv}\left(U_{E} \cap H\right) \subseteq U_{E}$, this topology is finer than the given topology on $E$. In particular, $\left(E, \mathcal{O}_{\text {conv }}\right)$ is a Hausdorff space. Obviously, the inclusion mapping $H \rightarrow\left(E, \mathcal{O}_{\text {conv }}\right)$ is continuous. Moreover, since $U_{E} \cap H=\operatorname{conv}\left(U_{E} \cap H\right) \cap H$, it is an embedding.

It remains to show that $\left(E, \mathcal{O}_{\text {conv }}\right)$ is a nuclear vector group. Therefore, let $p$ and $q$ be continuous pre-Hilbert seminorms on an open subspace $E_{0} \leq$ $E$ such that $\mathrm{d}_{k}\left(B_{p}, B_{q}\right) \leq \frac{1}{2} k^{-1}$ (see 2.2). Then

$$
d:=\sum_{k \in \mathbb{N}} \mathrm{d}_{k}\left(B_{p}, B_{q}\right)^{2} \leq \frac{1}{4} \cdot \frac{\pi^{2}}{6}<\frac{1}{2} .
$$

According to 3.20 of [2], we get

$$
\mathrm{d}_{k}\left(\operatorname{conv}\left(B_{p} \cap H\right), \operatorname{conv}\left(B_{q} \cap H\right)\right) \leq \frac{1}{1-d} \mathrm{~d}_{k}\left(B_{p}, B_{q}\right) \leq k^{-1},
$$

which completes the proof. 
LEMMA 5.3. Let $p$ and $q$ be pre-Hilbert seminorms on a vector space $E$ such that

$$
\frac{21}{2 \pi} \sum_{k \in \mathbb{N}} \mathrm{d}_{k}\left(B_{p}, B_{q}\right)<\frac{1}{4}
$$

and let $H$ be a subgroup of $E$. For every homomorphism $\chi: H \rightarrow \mathbb{T}$ satisfying $\chi\left(H \cap B_{q}\right) \subseteq\left\{e^{2 \pi i t}:|t| \leq 1 / 4\right\}$, there exists a linear homomorphism $f$ : $E \rightarrow \mathbb{R}$ such that $\left.e^{2 \pi i f}\right|_{H}=\chi$ and $f\left(B_{p}\right) \subseteq[-1 / 4,1 / 4]$.

Proof. This is Lemma 19.14(ii) in [1]; cf. also 8.1 in [2].

REMARKS 5.4. (i) The variety $\mathcal{V}_{1}$ is generated by all nuclear and all discrete vector spaces.

[Every discrete abelian group is a quotient group of a free abelian group and free abelian groups can be considered as subgroups of vector spaces.]

(ii) The variety $\mathcal{V}_{1}$ is generated by all special nuclear vector groups.

[According to 2.5, every special nuclear vector group belongs to $\mathcal{V}_{1}$. Conversely, according to 2.4(iv), every nuclear and every discrete vector space is a special nuclear vector group and hence the assertion follows from (i).]

LEMmA 5.5. A nuclear vector group $V$ belongs to $\mathcal{V}_{1}$ if and only if it is a special nuclear vector group.

Proof. One direction was proved in 2.5.

So let us prove the other implication. According to 5.4(i), there exist a nuclear vector space $E$, a family of discrete vector spaces $D_{j}(j \in J)$, a subgroup $H \leq E \times \prod_{j \in J} D_{j}$, and a quotient mapping $\varphi: H \rightarrow V$.

We denote the free abelian group over $H$ by $A(H)$; let $\eta: H \rightarrow A(H)$ be an injective mapping onto the basis $\eta(H)$ of $A(H)$. Further, we denote by $L(H)$ a vector space with basis $\eta(H)$ and we assume $A(H)$ to be a subgroup of $L(H)$. So we have

$$
H \stackrel{\eta}{\rightarrow} A(H) \stackrel{\tau}{\rightarrow} L(H)
$$

where $\tau$ denotes the canonical inclusion.

Now we endow $A(H)$ and $L(H)$ with the initial topology $\mathcal{O}$ induced by the inclusion $\tau$ and the linear operator $\psi$ :

$$
A(H) \stackrel{\tau}{\rightarrow} L(H) \stackrel{\psi}{\rightarrow}\left(E \times \prod_{j \in J} D_{j}\right) \times \mathbb{R}^{\mathcal{C}(H, \mathbb{R})}
$$

where

$$
\psi\left(\sum_{x \in H} \mu_{x} \eta(x)\right)=\left(\sum_{x \in H} \mu_{x} x,\left(\sum_{x \in H} \mu_{x} f(x)\right)_{f \in \mathcal{C}(H, \mathbb{R})}\right) \quad \text { for }\left(\mu_{x}\right) \in \mathbb{R}^{(H)}
$$

and $\mathcal{C}(H, \mathbb{R})$ denotes the space of continuous functions $H \rightarrow \mathbb{R}$. The space $\mathbb{R}^{\mathcal{C}(H, \mathbb{R})}$ is endowed with the product topology. 
Taking into consideration that $H$ is a completely regular space, there exists for every $0 \neq \sum \mu_{x} \eta(x) \in L(H)$ a continuous mapping $f: H \rightarrow \mathbb{R}$ such that $f(x)=\operatorname{sign}\left(\mu_{x}\right)$ for all $x$ such that $\mu_{x} \neq 0$. Hence

$$
\sum \mu_{x} f(x)=\sum\left|\mu_{x}\right|>0 .
$$

This shows that $\psi$ is injective.

According to $2.4(\mathrm{iv}),\left(E \times \prod_{j \in J} D_{j}\right) \times \mathbb{R}^{\mathcal{C}(H, \mathbb{R})}$ is a special nuclear vector group and so is $L(H)$.

Since $\psi(\tau(A(H)))$ is a subgroup of $H \times \mathbb{R}^{\mathcal{C}(H, \mathbb{R})}$ (and the topology on $A(H)$ is induced by the embedding $\left.A(H) \stackrel{\psi \circ \tau}{\longrightarrow} H \times \mathbb{R}^{\mathcal{C}(H, \mathbb{R})}\right)$, the projection

$$
\pi_{H}: A(H) \rightarrow H, \quad \sum k_{x} \eta(x) \mapsto \sum k_{x} x,
$$

is well defined and continuous. We now show that it is also open. Fix $U \in$ $\mathcal{U}_{H}(0)$ and a finite subset $C \subseteq \mathcal{C}(H, \mathbb{R})$, and let $\varepsilon>0$. Since

$$
\begin{aligned}
& \pi_{H}\left(\left\{\sum k_{x} \eta(x): \sum k_{x} x \in U \text { and }\left|\sum k_{x} f(x)\right|<\varepsilon \text { for all } f \in C\right\}\right) \\
& \supseteq\{x=x-0: x \in U \text { and }|f(x)-f(0)|<\varepsilon \text { for all } f \in C\}
\end{aligned}
$$

is a neighborhood of 0 in $H$, the projection $\pi_{H}$ is open.

This yields that the composition $\varphi \circ \pi_{H}$ is a continuous and open epimorphism. Let $\Phi: L(H) \rightarrow V$ be the (unique) linear extension of $\varphi \circ \pi_{H}$ and consider the following diagram:

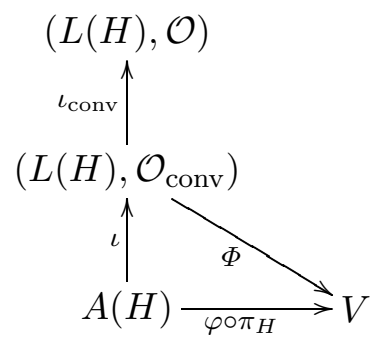

For $\mathcal{O}_{\text {conv }}$ as in 5.2 , the inclusion $\iota$ is an embedding, the identity $\iota_{\text {conv }}$ is continuous, and $\tau=\iota_{\text {conv }} \circ \iota$. In particular, by the definition of the 0 neighborhood basis in $\mathcal{O}_{\text {conv }}$ and since $V$ has a neighborhood basis of symmetric convex sets, the linear mapping $\Phi$ is continuous and, of course, open.

We fix a symmetric convex neighborhood $U \in \mathcal{U}_{V}(0)$. By the continuity of $\Phi$, there exists a continuous (with respect to $\mathcal{O}_{\text {conv }}$ ) seminorm $q$ on a subspace $L_{q}$ of $L(H)$ such that $\Phi\left(B_{q}\right) \subseteq U$. According to 5.2 and by assumption, $\mathcal{O}_{\text {conv }}$ and $\mathcal{O}$ induce the same topology on $A(H)$.

Since $(L(H), \mathcal{O})$ is a special nuclear vector group, there exists, due to $2.2(\mathrm{i})$ and (ii) and 2.4(iii), a sequence $\left(p_{n}\right)_{n \in \mathbb{N}_{0}}$ of pre-Hilbert seminorms defined on (the same) subspace $L_{p} \leq L(H)$ which are continuous with respect to $\mathcal{O}$ and which satisfy: 
(i) $A(H) \cap B_{q} \supseteq A(H) \cap B_{p_{0}}$,

(ii) $(21 /(2 \pi)) \sum_{k \in \mathbb{N}} \mathrm{d}_{k}\left(B_{p_{1}}, B_{p_{0}}\right)<1 / 4$, and

(iii) $\mathrm{d}_{k}\left(B_{p_{n+1}}, B_{p_{n}}\right) \leq k^{-1}$ for all $k, n \in \mathbb{N}$.

The subspace $L_{0}:=\left\langle A(H) \cap B_{q} \cap B_{p_{1}}\right\rangle_{\mathbb{R}} \leq L_{q} \cap L_{p}$ is open with respect to $\mathcal{O}_{\text {conv }}$ by the continuity of $\iota_{\text {conv }}$.

We wish to show

$$
L_{0} \cap B_{q} \supseteq L_{0} \cap B_{p_{1}} .
$$

Therefore, let $f: L_{0} \rightarrow \mathbb{R}$ be a linear functional such that $f\left(B_{q} \cap L_{0}\right) \subseteq I:=$ $[-1 / 4,1 / 4]$. Due to (i), we have $f\left(A(H) \cap B_{p_{0}} \cap L_{0}\right) \subseteq f\left(A(H) \cap B_{q} \cap L_{0}\right) \subseteq I$. Since

$$
\frac{21}{2 \pi} \sum_{k \in \mathbb{N}} \mathrm{d}_{k}\left(B_{p_{1}} \cap L_{0}, B_{p_{0}} \cap L_{0}\right) \stackrel{2.2(\mathrm{iii})}{\leq} \frac{21}{2 \pi} \sum_{k \in \mathbb{N}} \mathrm{d}_{k}\left(B_{p_{1}}, B_{p_{0}}\right)<\frac{1}{4},
$$

we can apply 5.3 to the character $\left.e^{2 \pi i f}\right|_{A(H) \cap L_{0}}$ and get a linear functional $\widetilde{f}: L_{0} \rightarrow \mathbb{R}$ which satisfies $\widetilde{f}\left(B_{p_{1}} \cap L_{0}\right) \subseteq I$ and $e^{2 \pi i \tilde{f}(x)}=e^{2 \pi i f(x)}$ for all $x \in A(H) \cap L_{0}$. This implies that $(f-\widetilde{f})\left(A(H) \cap L_{0}\right)$ is a subgroup of $\mathbb{Z}$. By assumption,

$$
(f-\widetilde{f})\left(A(H) \cap B_{q} \cap B_{p_{1}}\right) \subseteq(I-I) \cap \mathbb{Z}=\{0\},
$$

which shows that $\left.f\right|_{L_{0}}=\left.\widetilde{f}\right|_{L_{0}}$. In particular, we have $f\left(B_{p_{1}} \cap L_{0}\right) \subseteq I$.

This inclusion implies $\left(B_{q} \cap L_{0}\right)^{0} \subseteq\left(B_{p_{1}} \cap L_{0}\right)^{0}$ and an application of the Hahn-Banach theorem to the seminormed space $\left(L_{0},\left.q\right|_{L_{0}}\right)$ (cf. Chap. II, §3, Corollary 4 of [3]) yields $B_{p_{1}} \cap L_{0} \subseteq B_{q} \cap L_{0}$ (since $B_{q} \cap L_{0}$ is closed).

By the continuity of $\iota_{\text {conv }}$, the sets $B_{p_{n}} \cap L_{0}$ are neighborhoods of 0 with respect to $\mathcal{O}_{\text {conv }}$. They satisfy

$$
\mathrm{d}_{k}\left(B_{p_{n+1}} \cap L_{0}, B_{p_{n}} \cap L_{0}\right) \stackrel{2.2(\text { iii) }}{\leq} \mathrm{d}_{k}\left(B_{p_{n+1}}, B_{p_{n}}\right) \stackrel{\text { (iii) }}{\leq} 1 / k
$$

for all $k, n \in \mathbb{N}$ and $\left\langle B_{p_{n}} \cap L_{0}\right\rangle_{\mathbb{R}}=L_{p} \cap L_{0}=L_{0}$ for all $n \in \mathbb{N}$.

As a consequence of $(*)$ we get $\Phi\left(L_{0} \cap B_{p_{1}}\right) \subseteq \Phi\left(L_{0} \cap B_{q}\right) \subseteq U$. Since $\Phi$ is open, it is clear that the sequence $\left(\Phi\left(B_{p_{n}} \cap L_{0}\right)\right)_{n \in \mathbb{N}}$ of neighborhoods has the desired properties.

COROLlaRY 5.6. The nuclear vector group $\Sigma_{0}$ does not belong to $\mathcal{V}_{1}$ and hence $\mathcal{V}_{1}$ is properly contained in $\mathcal{V}_{2}$.

Proof. According to 2.12, $\Sigma_{0}$ is not a special nuclear group. As a consequence of the above lemma, $\Sigma_{0} \notin \mathcal{V}_{1}$. But $\Sigma_{0}$ is an element of $\mathcal{V}_{2}$, so the assertion follows.

Acknowledgments. I am very grateful to Helge Glöckner for pointing out an error (corrected in 5.6) in a manuscript on a related topic. His comments on the first draft of the present paper led to some simplification and to the elimination of inaccuracies. 
I also wish to express my thanks to the referee for the comments which are taken into account in the final version.

\section{References}

[1] L. Außenhofer, Contributions to the duality theory of abelian topological groups and to the theory of nuclear groups, Dissertationes Math. 384 (1999).

[2] W. Banaszczyk, Additive Subgroups of Topological Vector Spaces, Springer, Berlin, 1991.

[3] N. Bourbaki, Espaces vectoriels topologiques, Chap. 2, Hermann, Paris, 1953.

[4] K. H. Hofmann, S. A. Morris and M. Stroppel, Locally compact groups, residual Lie groups, and varieties generated by Lie groups, Topology Appl. 71 (1996), 63-91.

[5] T. Kōmura and Y. Kōmura, Über die Einbettung der nuklearen Räume in $(s)^{A}$, Math. Ann. 162 (1966), 284-288.

[6] S. A. Morris, Varieties of topological groups: a survey, Colloq. Math. 46 (1982), $147-165$.

[7] A. Pietsch, Nuclear Locally Convex Spaces, Springer, Berlin, 1972.

[8] - Operator Ideals, North-Holland, Amsterdam, 1980.

[9] S. Rolewicz, Metric Linear Spaces, PWN-Polish Sci. Publ., Warszawa, 1972.

[10] H. H. Schaefer, Topological Vector Spaces, Macmillan, New York, 1966.

Mathematisches Institut

Auf der Morgenstelle 10

D-72076 Tübingen, Germany

E-mail: lydia.aussenhofer@uni-tuebingen.de

Received October 13, 1999

Revised version December 27, 2000 